Auxiliary functions of the LISA laser link: ranging, clock noise transfer and data communication

This article has been downloaded from IOPscience. Please scroll down to see the full text article.

2011 Class. Quantum Grav. 28094008

(http://iopscience.iop.org/0264-9381/28/9/094008)

View the table of contents for this issue, or go to the journal homepage for more

Download details:

IP Address: 194.94.224.254

The article was downloaded on 08/07/2011 at 09:32

Please note that terms and conditions apply. 


\title{
Auxiliary functions of the LISA laser link: ranging, clock noise transfer and data communication
}

\author{
Gerhard Heinzel, Juan José Esteban, Simon Barke, Markus Otto, \\ Yan Wang, Antonio F Garcia and Karsten Danzmann \\ Max-Planck-Institute for Gravitational Physics (Albert-Einstein-Institute), Callinstrasse 38, \\ D-30167 Hanover, Germany \\ and \\ QUEST Centre for Quantum Engineering and Space-Time Research, Leibniz University of \\ Hanover, Hanover, Germany \\ E-mail: gerhard.heinzel@aei.mpg.de
}

Received 14 October 2010, in final form 14 January 2011

Published 18 April 2011

Online at stacks.iop.org/CQG/28/094008

\begin{abstract}
The Laser Interferometer Space Antenna (LISA) is required to reduce two important noise sources by post-processing on the ground using time-delay interferometry (TDI): phase noise of the on-board reference clocks and laser frequency noise. To achieve the desired suppression, the TDI algorithm needs measurements of the differential clock noise between any two spacecraft and inter-spacecraft ranging measurements with at least $1 \mathrm{~m}$ accuracy, which is beyond the precision of ground-based measurements for deep space missions. Therefore, we need on-board measurements by transmitting clock noise and ranging information between the spacecraft as auxiliary functions of the laser link. This paper reports our current experimental results in clock noise transfer and ranging for noise subtraction via post-processing as well as additional data transfer.
\end{abstract}

PACS numbers: $\quad$ 04.80.Nn, 07.60.Ly, 42.60.Fc, 95.55.Ym, 78.20.Jq, 42.60.Mi

(Some figures in this article are in color only in the electronic version)

\section{Introduction}

The Laser Interferometer Space Antenna (LISA), a joint ESA/NASA mission to be launched in the next decade, is designed to observe gravitational waves in the millihertz frequency band with a typical strain sensitivity of around $10^{-20} \mathrm{~Hz}^{-1 / 2}$ at $10 \mathrm{mHz}$ [1]. LISA uses heterodyne laser interferometry to measure the displacement fluctuations between six free floating test masses enclosed in three separate spacecraft with a sensitivity of $\approx 10 \mathrm{pm} \mathrm{Hz}^{-1 / 2}$. Laser beams at $\lambda=1064 \mathrm{~nm}$ are exchanged between the spacecraft and on each spacecraft a local laser is interfered with by the incoming laser light influenced by gravitational waves. The 
phase of the resulting carrier-to-carrier beat note is then measured with an allocated noise budget of $1 \mu$ cycle $\mathrm{Hz}^{-1 / 2}$.

The orbits of the LISA formation are designed to keep the arm lengths as stable as possible in the solar system environment, with the resulting arm length variation of $\leqslant 1 \% \approx 50000 \mathrm{~km}$ [2] with line-of-sight velocities below $\pm 15 \mathrm{~m} \mathrm{~s}^{-1}$ [3]. For precision interferometers, this is, however, still an enormous arm length imbalance. Additionally the beat notes have frequencies of up to $20 \mathrm{MHz}$ due to the Doppler shifts and necessary frequency offsets in the phase locks.

As a consequence, the LISA sensitivity would be limited by two main noise sources: the first one is given by the phase noise of the spacecraft clocks. A timing stability of $50 \mathrm{fs} \mathrm{Hz}^{-1 / 2}$ would be needed to achieve the required phase sensitivity for a beat note at $20 \mathrm{MHz}$, and such stability is not achievable for any space-qualified clock. The second noise source enters due to the unequal arm length configuration. The laser frequency noise, somewhere between 30 and $1000 \mathrm{~Hz} / \mathrm{Hz}^{-1 / 2}$ after laser pre-stabilization, directly couples into the phase measurement.

One can remove both noise sources using a post-processing technique on the ground called time-delay interferometry (TDI) [4-6]. However, this technique requires additional measurements: the clock phase noise, the absolute distance between the spacecraft to about $1 \mathrm{~m}$ [7], and the clock offset between spacecraft to a few nanoseconds. These measurements are performed by applying two different phase modulations to the laser link. To measure the clock noise, the phase noise of each of the three spacecraft master clocks is multiplied by an integer factor and modulated as high-frequency $(\mathrm{GHz})$ phase modulation sidebands onto each laser link using $10 \%$ of the light power. After interference between local and incoming lasers, the phase measurement of the resulting sideband-to-sideband beat note contains the amplified clock noise information necessary to remove the clock noise by TDI.

Absolute inter-spacecraft distances are determined with a pseudo-random noise (PRN) phase modulation on each laser carrier using $0.1-1 \%$ of the light power [8-10]. The distance is measured via correlation of the demodulated carrier phase with a local copy of the original PRN code. An important benefit of such a modulation is the possibility of additional data encoding on top of the PRN codes to enable inter-spacecraft communication. A direct measurement of the clock offsets between the three spacecraft to a few nanoseconds is an automatic by-product of this technique.

In this paper, we present our experimental results toward a laboratory demonstration of ranging, data communication and clock noise transfer for LISA. Section 2 formulates the main LISA data streams and gives an overview of the TDI algorithm. The phase stability of necessary components in the frequency distribution system is discussed in section 4 . Section 5 shows the experimental setup built to test ranging and clock noise transfer performance. Additionally, we also describe the data pre-processing techniques to extract optimal estimates for clock offsets and spacecraft distances with Kalman-like filters.

\section{Time-delay interferometry}

The challenge of TDI is to remove the two main noise sources (laser phase noise and clock noise) while preserving the gravitational wave signal. The basic idea of TDI is to combine time-shifted phase measurements from the three satellites in post-processing on the ground to synthesize a virtual interferometer with equal arm lengths (see [11] and references therein). We briefly review the TDI equations here, which are presently being rewritten for the current baseline layout of the optical benches $(\mathrm{OB})$. This work also includes a more detailed discussion of clock noise and the signs of the beat notes and will be published soon. To formulate the equations of the LISA-like data streams, we follow the notation shown in figure 1 and introduce 


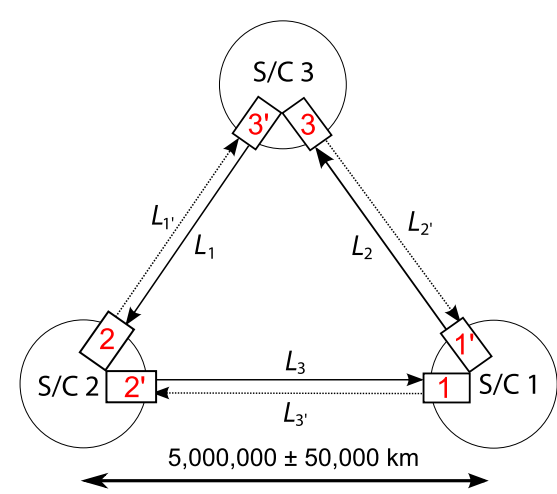

Figure 1. Schematic of the LISA constellation with TDI notation. The resulting Doppler frequency shift due to arm length variation is about $20 \mathrm{MHz}$.

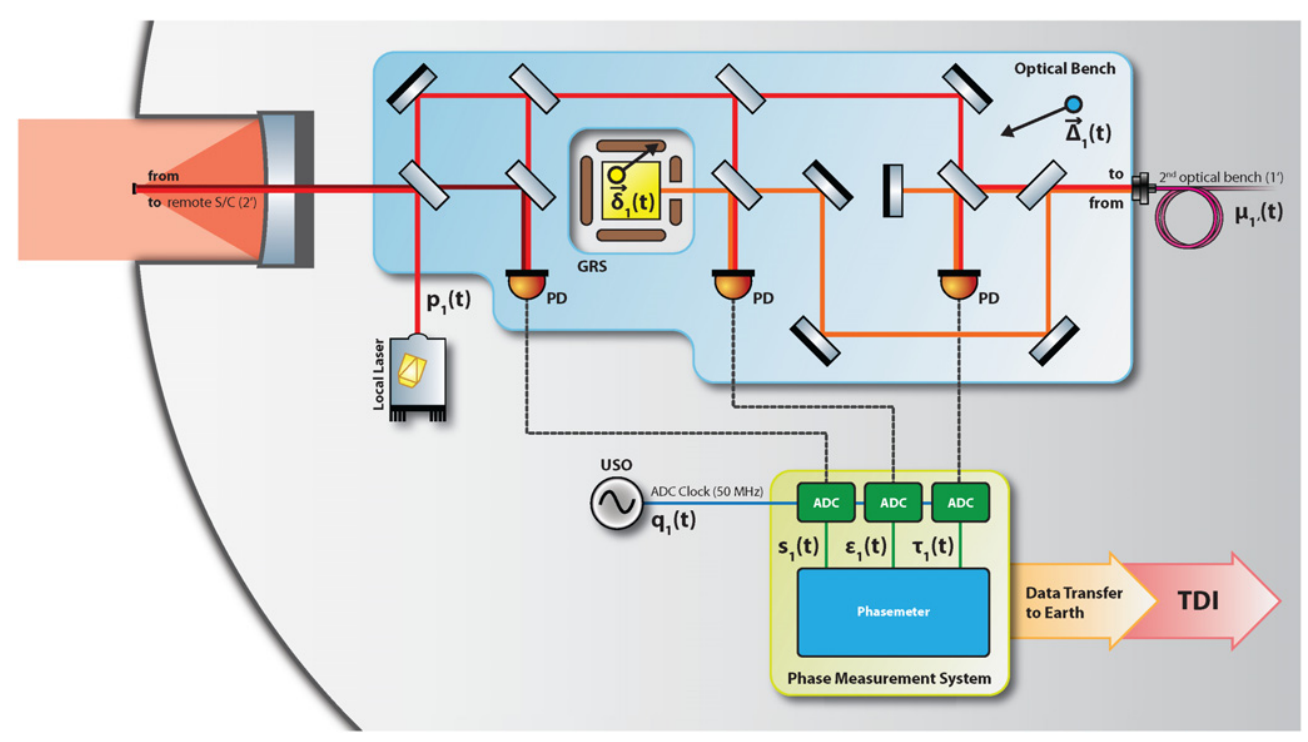

Figure 2. Simplified sketch of the LISA measurement system.

a time-delay operator $\mathcal{D}$ acting on a continuous function $f(t)$ via

$$
\mathcal{D}_{k} f(t)=f\left(t-L_{k}\right), \quad \mathcal{D}_{k} \mathcal{D}_{j} f(t)=f\left(t-L_{j}-L_{k}\right),
$$

where $c=1$ is used and the indices can have the values $1,1^{\prime}, 2,2^{\prime}, 3,3^{\prime}$.

In the following, TDI 1.5 (called modified TDI) is used, in which the arm lengths are treated as constant but the Sagnac effect due to the cartwheel motion of the satellite constellation does affect the measurement. Thus, the direction of the light travel is important: $L_{i} \neq L_{i^{\prime}}$.

We now show the main equations for the measured data, referring to the highly simplified sketch of the LISA measurement system (optical bench 1) shown in figure 2.

Three main measurements take place on each OB: the science interferometer signal $s_{1}(t)$ measures on the left photodiode (PD) the beat note between the incoming laser beam from 
the distant $\mathrm{S} / \mathrm{C}$ and the local transmitted laser, which is used as local oscillator. The test mass motion is measured in the signal $\varepsilon_{1}(t)$ (middle PD) using the two locally available strong laser sources, the transmitted laser of the bench under discussion and the laser of the adjacent bench $1^{\prime}$, which is delivered through a 'backlink' fiber with additional phase noise $\mu_{1^{\prime}}(t)$. The same two laser sources are used for the reference interferometer with the signal $\tau_{1}(t)$ (right PD) but without the test mass. Ideally, these signals are given by

$$
\begin{aligned}
& s_{1}(t)=h_{1}(t)+\mathcal{D}_{3} p_{2^{\prime}}(t)-p_{1}(t)+N_{1}^{s}(t) \\
& \tau_{1}(t)=p_{1^{\prime}}(t)-p_{1}(t)+\mu_{1^{\prime}}(t)+N_{1}^{\tau}(t) \\
& \varepsilon_{1}(t)=p_{1^{\prime}}(t)-p_{1}(t)+\mu_{1^{\prime}}(t)-\frac{4 \pi}{\lambda_{1}} \vec{n}_{3} \cdot \vec{\delta}_{1}(t)+N_{1}^{\varepsilon}(t),
\end{aligned}
$$

where $h_{1}(t)$ is the gravitational wave signal measured on OB $1, p_{1}$ is the phase noise of the local oscillator on OB 1 and $p_{2^{\prime}}$ is the laser phase noise of the laser mounted on OB 2. The distant laser noise is time-shifted due to the travel time along $L_{3}$. $\lambda_{1}$ denotes the wavelength of the laser and $\vec{\delta}_{1}$ the test mass motion on OB 1 . Hence, $-\frac{4 \pi}{\lambda_{1}} \vec{n}_{3} \cdot \vec{\delta}_{1}$ represents the phase shift along $L_{3}$ due to the test mass motion. $N_{1}^{s, \varepsilon, \tau}(t)$ combines additional noise sources like optical bench motion, shot noise and electronic noise of the photodiodes and the phasemeter. The signals on the other optical benches are similar.

It was shown in [12] that the laser phase noise can be removed from the above equations by combining and time-shifting the data of all spacecraft. One laser phase noise free combination is given by

$$
\begin{aligned}
X(t)=\left(\mathcal{D}_{2^{\prime}} \mathcal{D}_{2} \mathcal{D}_{3} \phi_{2^{\prime}}+\mathcal{D}_{2^{\prime}} \mathcal{D}_{2} \phi_{1}+\mathcal{D}_{2^{\prime}} \phi_{3}+\phi_{1^{\prime}}\right) \\
-\left(\mathcal{D}_{3} \mathcal{D}_{3^{\prime}} \mathcal{D}_{2^{\prime}} \phi_{3}+\mathcal{D}_{3} \mathcal{D}_{3^{\prime}} \phi_{1^{\prime}}+\mathcal{D}_{3} \phi_{2^{\prime}}+\phi_{1}\right),
\end{aligned}
$$

called the TDI-X combination (or TDI Michelson); it synthesizes a virtual Michelson interferometer. Here, the $\phi$ are appropriate combinations of the above $s, \tau, \varepsilon$-signals.

Application of the delay operators $\mathcal{D}_{k}$ implies that both the spacecraft separations and the time when each measurement was taken are known to sufficient accuracy (roughly $\mathrm{m}$ and ns).

Each satellite carries an ultrastable oscillator (USO) as a master clock which is also used to trigger the ADC sampling process in the phasemeter. This USO furthermore has clock noise $q(t)$ that affects the measured data. Hence, the above equations for OB 1 have to be rewritten as

$$
\begin{aligned}
& s_{1}(t)=h_{1}(t)+\mathcal{D}_{3} p_{2^{\prime}}(t)-p_{1}(t)+\alpha_{1} q_{1}(t)+N_{1}^{s}(t) \\
& \tau_{1}(t)=p_{1^{\prime}}(t)-p_{1}(t)+\beta_{1} q_{1}(t)+\mu_{1^{\prime}}(t)+N_{1}^{\tau}(t) \\
& \varepsilon_{1}(t)=p_{1^{\prime}}(t)-p_{1}(t)+\beta_{1} q_{1}(t)+\mu_{1^{\prime}}(t)-\frac{4 \pi}{\lambda_{1}} \vec{n}_{3} \cdot \vec{\delta}_{1}(t)+N_{1}^{\varepsilon}(t)
\end{aligned}
$$

where $L$ must be obtained from the noisy range measurements $\widehat{L}$, and $\alpha_{1}=\frac{\Delta f_{s_{1}}}{f_{\mathrm{ADC}}}$ and $\beta_{1}=\frac{\Delta f_{\tau_{1}}}{f_{\mathrm{ADC}}}=\frac{\Delta f_{\varepsilon_{1}}}{f_{\mathrm{ADC}}}$ are the clock noise conversion factors for the ADC sampling taking place at $f_{\mathrm{ADC}} \approx 50 \mathrm{MHz}$. The beat note frequencies $\Delta f$ are between 2 and $20 \mathrm{MHz}$ and vary with time.

There is not yet enough information to remove clock noise from the data streams. Hence, the modulation scheme described in [13] is applied to obtain additional sideband-sideband measurements with amplified clock noise. Subsequently, a three-step procedure can be applied to remove the main noise sources.

(i) Redefine the LISA data streams to remove primed laser noise $p_{1^{\prime}}, p_{2^{\prime}}$ and $p_{3^{\prime}}$, thus effectively dealing with only three lasers. 
(ii) Define a master reference clock, e.g. $q_{1}(t)=0$ and use the additional sideband information to cancel clock noise.

(iii) Remove the laser phase noise via TDI.

It is possible to show analytically that this scheme works for TDI 1.5. The experimental demonstration was recently performed and published in $[4,6]$.

\section{Baseline architecture and requirements}

The present architecture foresees the following ingredients to achieve all functions described above:

- A fiber-coupled phase modulator (EOM) that imprints both the $\mathrm{GHz}$ sidebands and the PRN codes on each laser beam before it enters the optical bench.

- A frequency distribution system that coherently derives the following signals from the USO aboard each spacecraft: The GHz modulation sidebands at an integer multiple of the basic frequency, the ADC sampling clock and the phasemeter pilot tone. One critical property is the phase stability between the ADC clock/pilot tone (around 50 or $60 \mathrm{MHz}$ ) and the GHz sidebands on the light that leaves the telescope. Since there is no easy way to detect the optical $\mathrm{GHz}$ sideband phase with respect to the carrier and thus perform a measurement or feedback correction, one has to rely on and experimentally prove the phase stability of each component in that chain. Some results have been previously published, e.g. on the dispersion of laser amplifiers $[14,15]$, and others are reported in the following section.

- The PRN code generation and recovery in the phasemeter. The performance of the whole ranging subsystem, including data transfer, can also be tested on the ground. Requirements are a ranging accuracy below $1 \mathrm{~m}$ at an update rate of $1 \mathrm{~Hz}$, and a data rate of $15 \mathrm{kbits} \mathrm{s}^{-1}$ with sufficiently low bit error rate. Some results have been published in $[8,9,16]$, and an update with the latest results is given below.

- Algorithms to combine the various measurements to an optimal estimate of clock offset and the spacecraft distance. The problems and first results are discussed below.

In addition, none of these functions may disturb the primary science measurement, which is the phase of the carrier-carrier beat note with a total noise budget for the single link of $10 \mathrm{pm} \mathrm{Hz}-1 / 2$. All requirements apply for Fourier frequencies between $3 \mathrm{mHz}$ and $1 \mathrm{~Hz}$ and relax as $f^{-2}$ between 3 and $0.1 \mathrm{mHz}$.

\section{Phase fidelity of the frequency distribution subsystem and clock tone transfer chain}

\subsection{Multipliers (synthesizers) versus dividers}

To maintain a coherent and stable phase relationship between a $\mathrm{MHz}$ and a $\mathrm{GHz}$ signal, one could either upconvert the $\mathrm{MHz}$ signal by means of a frequency synthesizer or digitally divide a GHz signal down to the $\mathrm{MHz}$ range. We tested both options for their phase stability.

The fractional-N synthesizer under test (custom-built by Ingenieurbüro Gronefeld, using a synthesizer chip available as space-qualified version) was operated with a $10 \mathrm{dBm}$ sinusoidal input signal and provided a $13 \mathrm{dBm}$ output ( $2 \mathrm{GHz} \pm 25 \mathrm{MHz}$ tuneable in $3 \mathrm{~Hz}$ steps). It was tested by upconverting the same $50 \mathrm{MHz}$ signal by two identical synthesizers to a frequency of $2 \mathrm{GHz}$ and subsequently mixing the output signals down to $1 \mathrm{MHz}$ with a common reference (figure 3, top left). 

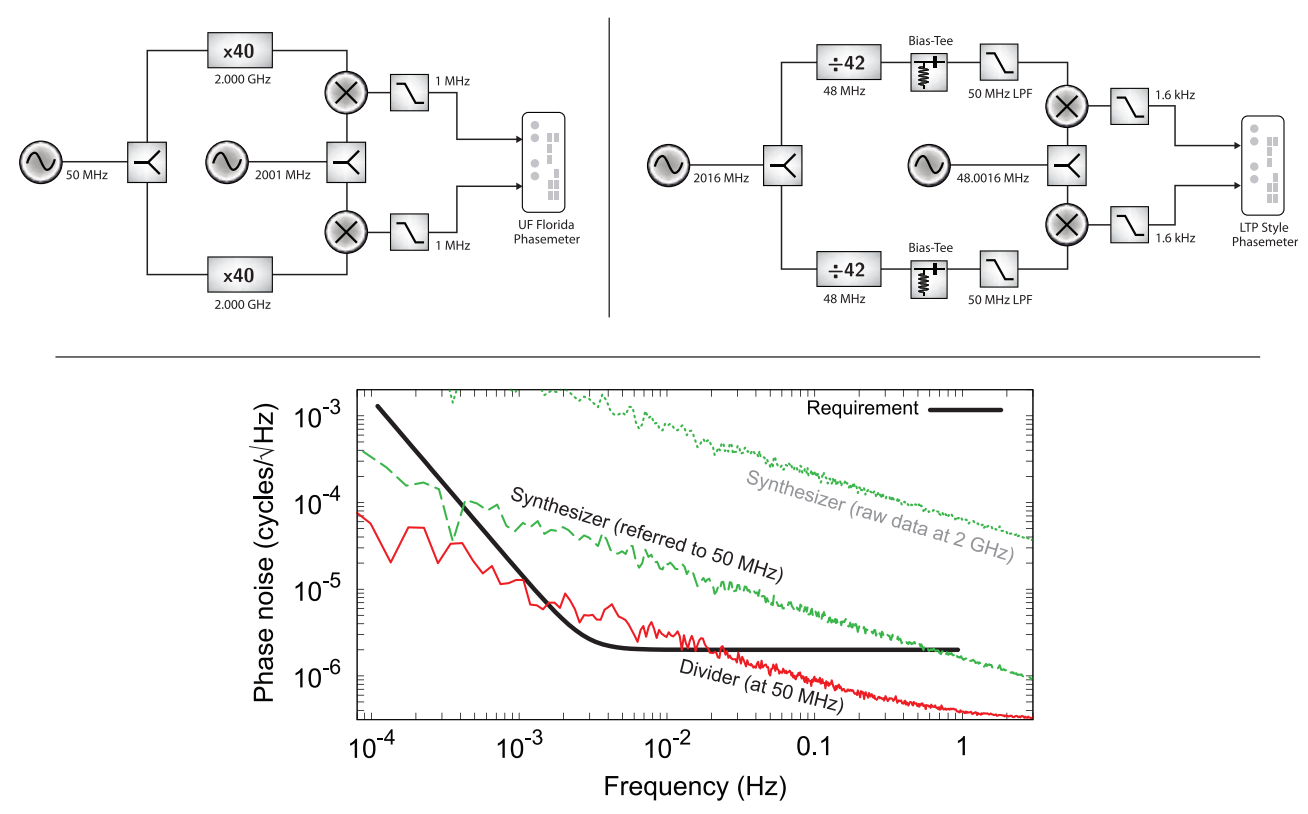

Figure 3. Top left: measurement setup for measuring the differential phase noise of any two synthesizers. Top right: measurement setup for measuring the differential phase noise of any two dividers. Bottom: comparison of differential phase noise introduced by synthesizers (top green curve: measured noise at $2 \mathrm{GHz}$; bottom green curve: excess noise after TDI) and dividers, respectively.

The difference in the phase of the output signals was measured with a LISA style phasemeter prototype developed by the University of Florida [17] and reveals the differential phase noise between the two synthesizers. This measurement was done with collaboration between the Albert Einstein Institute and the University of Florida during a visit in Florida by one of the authors (SB).

The digital divider under test (evaluation board UXN14M9PE by Centellax, available as space-qualified version) was operated with a $0 \mathrm{dBm}$ sinusoidal input signal and provided a $4 \mathrm{dBm}$ square-wave output frequency divided by an integer between 8 and 511 . It was tested by dividing the same $2.016 \mathrm{GHz}$ signal by means of two identical dividers down to a frequency of $48 \mathrm{MHz}$ and subsequently mixing the output signals down to $1.6 \mathrm{kHz}$ with a common reference (figure 3, top right). The difference in the phase of the two output signals was measured with a LTP style phasemeter developed by the Albert Einstein Institute, Hanover [18], and again reveals the differential phase noise between the two dividers.

While the differential phase noise of the dividers did comply with the LISA requirements for almost the complete frequency spectrum without any extra effort, the synthesizer was adding a significant amount of excess phase noise to the signal (cf figure 3, bottom). This high noise might be due to the correlation between the input amplitude and the output phase. These two quantities probably couple at the synthesizer's phase detector. The coupling coefficient was measured to be $0.2 \mathrm{rad} / \mathrm{V}_{\mathrm{pp}}$ in the differential and $4.4 \mathrm{rad} / \mathrm{V}_{\mathrm{pp}}$ in the individual signals. This requires a $3.1 \times 10^{-3} \mathrm{~V}_{\mathrm{pp}} \mathrm{Hz}^{-1 / 2}$ amplitude stability at $10 \mathrm{dBm}$ signal level which was not met by our signal generator. However, since the amplitude-phase relationship might not be the only limiting noise source, more tests are necessary before we can give a final statement on the synthesizers' usability. 

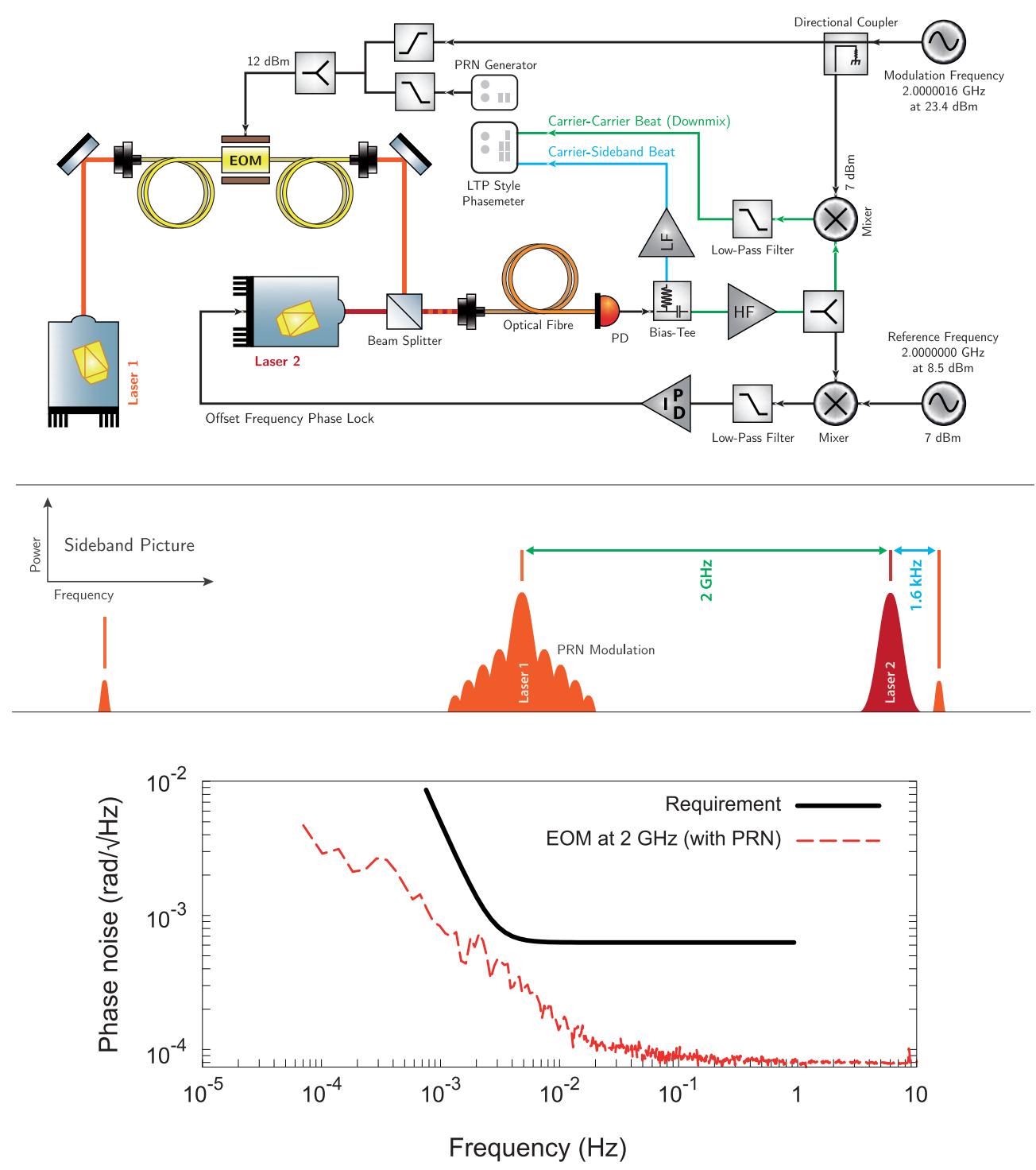

Figure 4. Top: setup for measuring the phase fidelity of a single EOM sideband. Center: sideband picture of the beat notes as detected by the photodiode. Bottom: measured phase noise of one single sideband for the Northrop Grumman LITEF GmbH EOM under test.

\subsection{Electro-optic modulator phase fidelity}

The electro-optic modulator (EOM) under test (provided by Northrop Grumman LITEF $\mathrm{GmbH}$, available as space-qualified version) has an efficiency of $0.7 \mathrm{rad} \mathrm{V}^{-1}$ at $2 \mathrm{GHz}$ and was operated at $12 \mathrm{dBm}$ to set the power in the generated sidebands to $10 \%$ of the carrier power (modulation index of 0.6). As done previously for tests on a different EOM [13] we used a setup for measuring the phase characteristics of one single EOM sideband. As shown in figure 4 (top), two lasers were offset phase-locked to $2 \mathrm{GHz}$ with one beam sent through an EOM driven by a signal at $2 \mathrm{GHz}+1.6 \mathrm{kHz}$ frequency. Both beams were heterodyned 


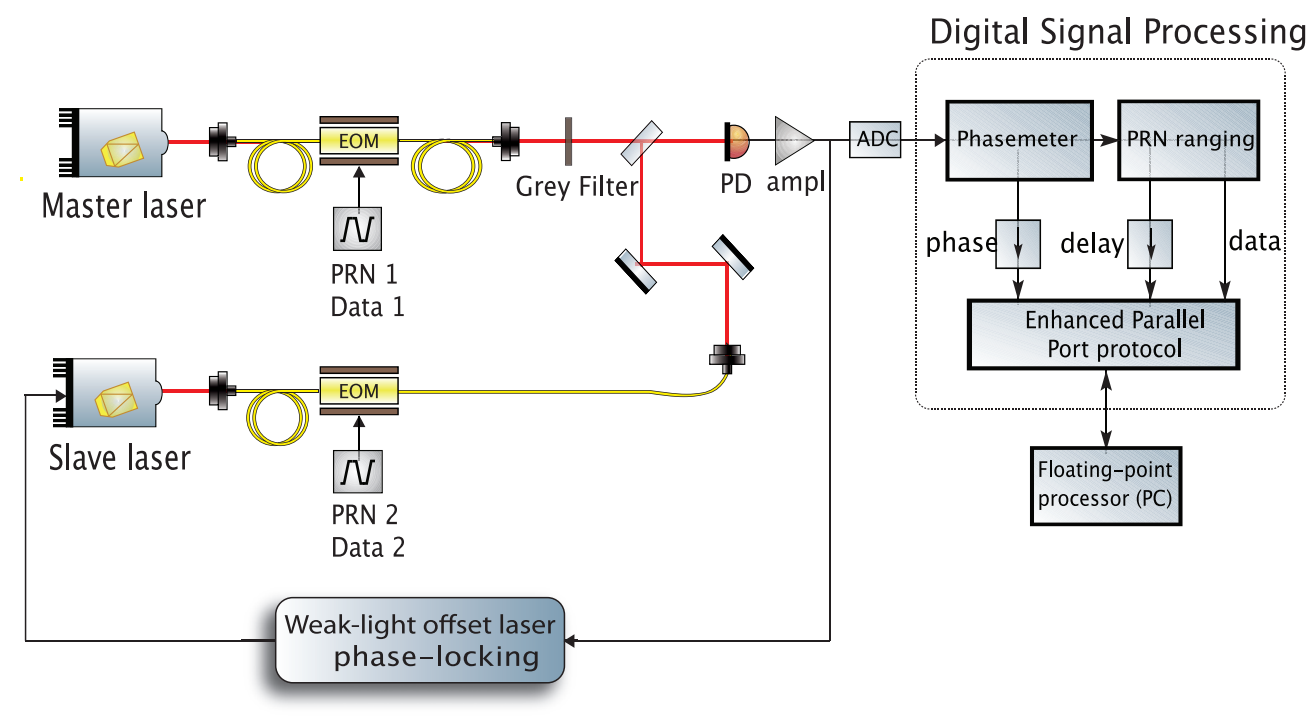

Figure 5. General schematic of the experimental setup used to test the laser modulation scheme.

and sent to a photodiode. In addition, we combined the $2 \mathrm{GHz}+1.6 \mathrm{kHz}$ modulation signal with a PRN code (cf section 5) to show that neither the PRN code itself nor the combiner electronics (mini-circuits components: Power Combiner ZX10R-14+, DC-10 GHz; High Pass filter VHP-16, pass band 1.9-2.7 GHz; and Low Pass filter VLFX-825, pass band DC-825 $\mathrm{MHz}$ ) interfere with the phase stability.

The phase of the $1.6 \mathrm{kHz}$ sideband-carrier beat note was measured. The carrier-carrier beat note was mixed down, low-pass filtered and the phase of the resulting $1.6 \mathrm{kHz}$ signals was also measured (figure 4, center). The difference of both measurements represents the noise of a single EOM sideband introduced by the EOM itself. Our measurements presented in figure 4 (bottom) show that the EOM under test complies with the LISA requirement.

\section{Ranging and data transfer}

An optical experiment was built to test the performance of clock noise transfer, PRN ranging and data transfer. The test-bed provides representative signals of the LISA interferometry system (see figure 5). A free-running master laser is phase modulated with a PRN code of time-varying phase running at $1.5 \mathrm{MHz}$ and additionally encoded with data at $12 \mathrm{kbps}$. A code generator in the FPGA drives the EOM to modulate the laser carrier using $1 \%$ of the light power. After interference between the incoming and the offset phase-locked local laser, a single element InGaAs photodetector with transimpedance amplifier senses the beat note. It is digitized and processed in our prototype of a LISA-like phasemeter [19]. In order to test the ranging performance, the light power of the master laser is attenuated to $100 \mathrm{pW}$ using gray filters, and the slave laser is modulated with a different PRN code.

Figure 6 shows the ranging accuracy as the standard deviation of the estimated delay for different measurement rates. The tracked signal is a time-varying PRN code equivalent to a relative velocity higher than $\approx 20 \mathrm{~m} \mathrm{~s}^{-1}$. The ranging measurements were obtained under normal- and weak-light environments for comparison. Experimental results demonstrate an 


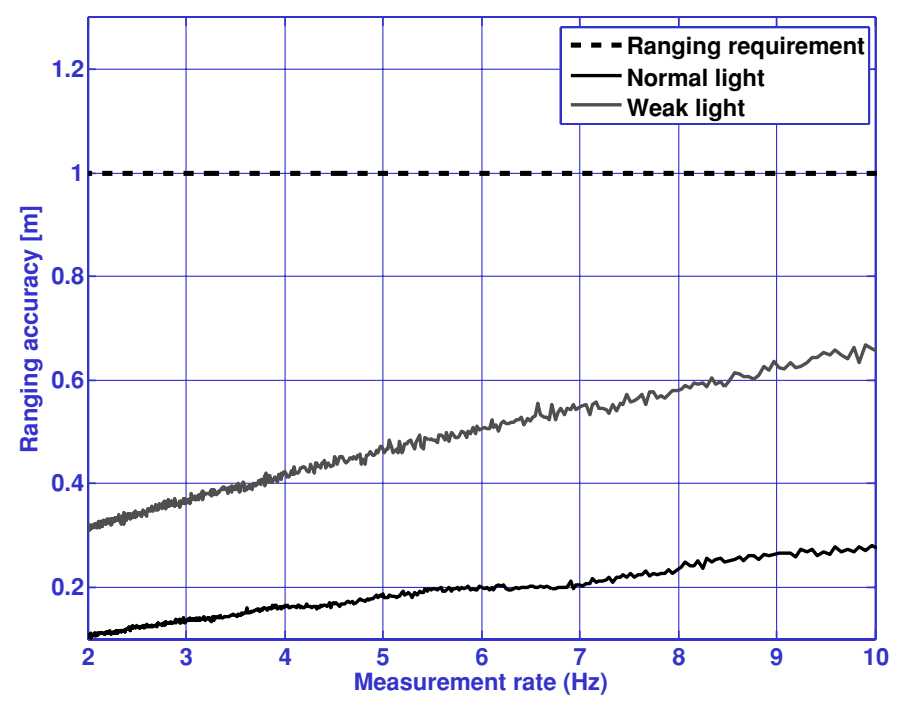

Figure 6. Standard deviation of the optical ranging measurements in the presence of LISAlike noise sources, i.e. interference with a second PRN, data encoding and electrically simulated inter-spacecraft velocity.

rms ranging noise of $35 \mathrm{~cm}$ at $3 \mathrm{~Hz}$ with a bit error rate (BER) better than $6 \times 10^{-4}$, which can be further improved by error-correction coding.

The ranging accuracy is mainly limited by shot noise in the weak-light conditions. Nevertheless, even under these conditions the implemented architecture is capable of achieving the accuracy requirement for the LISA mission.

\subsection{Data analysis techniques for optimal filtering}

In the first processing step on the ground, the received code phase, interpreted as time stamps, must be combined with a dynamic model of the orbit evolution to extract the time-variable clock offsets and distances and synthesize a virtual common constellation clock [20]. An optimal filter (Kalman-like) is being investigated for this purpose. It is non-standard because the time when each measurement was taken is affected by the clock offset, which is itself part of the unknown state vector.

Figure 7 shows the preliminary performance of the optimal filter on $320 \mathrm{~s}$ of simulated measurements. The blue points in the two pictures show the measurement noise in pseudo-ranging measurements (DC values of clock bias are not included) and the Doppler measurements, respectively. The red points are the residual noise after filtering. Note that the optimal filter can significantly reduce the noise level in both the arm lengths measurements and Doppler measurements. More details about this will be published soon. Further investigations will include the unknown DC values of clock bias and investigate backward filtering ('smoothing'), iterative improvement of the solutions and interpolation of the data streams to arbitrary times of the common virtual clock. 

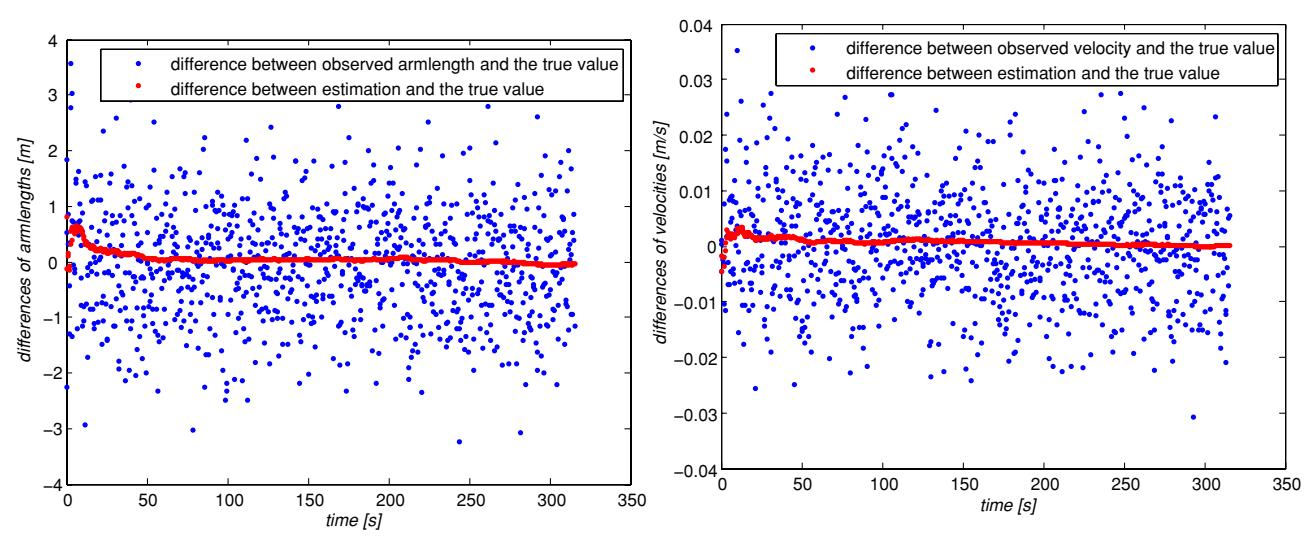

Figure 7. Noise suppression in the arm length and longitudinal velocity measurements. Scattered: raw observations. Smooth: filtered estimates.

\section{Conclusion and future work}

This paper presented our current investigations toward an experimental demonstration of laser frequency noise and clock jitter subtraction using the TDI algorithm. In order to apply TDI, precise ranging and clock phase noise measurements need to be verified under LISA-like conditions and with the required sensitivity. Therefore, a heterodyne interferometer was built as a test bed for PRN ranging and clock noise transfer. Experimental results fulfilled all requirements with margins. Further work is in progress to put the pieces together and eventually demonstrate the complete generation of TDI variables in circumstances as close to the LISA situation as ground-based experiments allow.

\section{Acknowledgments}

We gratefully acknowledge the support by Deutsches Zentrum für Luft-und Raumfahrt (DLR) (reference 50 OQ 0501 and 50 OQ 0601) and thank the German Research Foundation for funding the Cluster of Excellence QUEST_Centre for Quantum Engineering and SpaceTime Research.

\section{References}

[1] The LISA Study Team 1998 Laser Interferometer Space Antenna for the detection and observation of gravitational waves Pre-phase A report Max-Planck Institute for Quantum Optics (http://edoc.mpg.de/335189)

[2] Dhurandhar S V, Nayak K Rajesh, Koshti S and Vinet J-Y 2005 Fundamentals of the LISA stable flight formation Class. Quantum Grav. 22481

[3] LISA 2009 Laser Interferometer Space Antenna: sciencecraft description, LISA-SC-DD-0001, NASA Goddard Space Flight Center (http://lisa.gsfc.nasa.gov/Documentation/LISA-SC-DD-0001.pdf)

[4] de Vine G, Ware B, McKenzie K, Spero R E, William M, Klipstein and Shaddock D A 2010 Experimental demonstration of time-delay interferometry for the laser interferometer space antenna Phys. Rev. Lett. 104211103

[5] Massimo T, Shaddock D, Sylvestre J and Armstrong J 2003 Implementation of time-delay interferometry for LISA Phys. Rev. D 67122003

[6] Mitryk S, Wand V and Mueller G 2010 Verification of time-delay interferometry techniques using the university of florida LISA interferometry simulator Class. Quantum Grav. 27084012 
[7] LISA Frequency Control Study Team 2009 ESA Report No LISA-JPL-TN-823 at press

[8] Esteban J J, Bykov I, Garcia Marin A F, Heinzel G and Danzmann K 2009 Optical ranging and data transfer development for LISA J. Phys.: Conf. Ser. 154012025

[9] Esteban J J, Garcia Marin A F, Eichholz J, Peinado A M, Bykov I, Heinzel G and Danzmann K 2010 Ranging and phase measurement for LISA J. Phys: Conf. Ser. 228012045

[10] Sutton A, McKenzie K, Ware B and Saddock D 2010 Laser ranging and communication for LISA Opt. Express 1820759

[11] Tinto M, Estabrook F B and Armstrong J W 2004 Time delay interferometry with moving spacecraft arrays Phys. Rev. D 69082001

[12] Tinto M, Estabrook F B and Armstrong J W 1999 Time-delay interferometry for space-based gravitational wave searches Astrophys. J. 527 814-26

[13] Barke S, Troebs M, Sheard B, Heinzel G and Danzmann K 2010 EOM sideband phase characteristics for the spaceborne gravitational wave detector LISA Appl. Phys. B 98 No $133-9$

[14] Tröbs M, Barke S, Theeg Th, Kracht D, Heinzel G and Danzmann K 2010 Differential phase-noise properties of a ytterbium-doped fiber amplifier for the laser interferometer space antenna Opt. Lett. 35 435-7

[15] Tröbs M, Barke S, Möbius J, Engelbrecht M, Theeg Th, Kracht D, Sheard B, Heinzel G and Danzmann K 2010 Fiber modulators and fiber amplifiers for LISA J. Phys.: Conf. Ser. 228012042

[16] Esteban J J, Garcia Marin A F, Bykov I, Heinzel G and Danzmann K 2009 Free-space laser ranging and data communication 6th Workshop on Positioning, Navigation and Communication IEEE Conf. Proc. 275-81

[17] Cruz R J et al 2006 The LISA benchtop simulator at the university of florida Class. Quantum Grav. 23 S751

[18] Heinzel G et al 2004 The LTP interferometer and phasemeter Class. Quantum Grav. 21 S581

[19] Bykov I, Esteban J J, Garcia Marin A F, Heinzel G and Danzmann K 2009 LISA phasemeter development: advanced prototyping J. Phys.: Conf. Ser. 154012017

[20] Shaddock D A, Ware B, Spero R E and Vallisneri M 2004 Postprocessed time-delay interferometry for LISA Phys. Rev. D 70081101 